\title{
Phase diagram of hard asymmetric dumbbell particles
}

\author{
Kristina Milinković, ${ }^{1, *}$ Matthew Dennison, ${ }^{2}$ and Marjolein Dijkstra ${ }^{1, \dagger}$ \\ ${ }^{1}$ Soft Condensed Matter, Debye Institute for Nanomaterials Science, Utrecht University, Princetonplein 5 , \\ 3584 CC Utrecht, The Netherlands \\ ${ }^{2}$ Department of Physics and Astronomy, Vrije Universiteit, De Boelelaan 1081, 1081 HV Amsterdam, The Netherlands
}

(Received 4 December 2012; published 13 March 2013)

\begin{abstract}
Using Monte Carlo simulations and free energy calculations, we study the phase behavior of hard asymmetric dumbbell particles with a constituent sphere diameter ratio of 0.5 . We find a rich phase behavior with isotropic fluid, rotator, and periodic $\mathrm{NaCl}$-based and both periodic and aperiodic $\mathrm{CrB}$-based crystalline phases. The rotator phases found to be stable in this study are similar to those found in systems of snowman-shaped and dumbbell particles and we investigate the behavior of these phases by comparing their stability ranges, and by looking at the orientational reorganization of particles. We also find that the $\mathrm{NaCl}$-based crystalline phase can expand its range of stability by undergoing a slight modification which allows it to pack better. Finally, we see that reducing the sphere separation results in the aperiodic crystalline phases becoming destabilized as compared to the phase behavior of snowman-shaped particles.
\end{abstract}

DOI: 10.1103/PhysRevE.87.032128

PACS number(s): 05.40.-a, 07.05.Tp, 81.30.-t, 05.10.Ln

\section{INTRODUCTION}

With the continual progress of techniques for synthesizing novel anisotropic colloidal particles comes the need to advance our understanding of the phase behavior of these systems accordingly. It is now not only possible to synthesize colloids with simple shapes such as discs [1], rods [2], and cubes [3], but also more complex morphologies such as raspberry-like colloids [4] and octopods [5]. However, predicting the collective system behavior is not trivial, even for simple geometries.

Perhaps the simplest anisotropic particle, which has been the focus of many theoretical [6-8] and simulation [9-15] studies, is the dimer. Dimer particles are not only fundamentally interesting, e.g., as a model for diatomic molecules, but also have practical applications, e.g., in the production of colloidal crystals with useful optical properties [16,17]. Dimers consist of two connected spheres, and they can be synthesized with a range of different constituent sphere diameter ratios and separations $[4,18-25]$. This ability to vary both the diameter ratio of the constituent spheres and their separation results in a vast parameter space, even for dimer particles interacting only via excluded volume.

In a recent publication [15] we used computer simulations to map out the phase diagram of hard tangential dimer (snowman-shaped) particles with varying constituent sphere diameter ratios. We found that the stable structures at high densities are colloidal crystals analogous to the best packed structures for equimolar binary hard-sphere mixtures. Specific orientational organization of particles within a crystal was found to have no effect on the packing, and as such crystals with aperiodic ordering of particles are stabilized by the degeneracy entropy. In these tangential systems, the degeneracy entropy also stabilizes additional less well-packed crystalline phases at intermediate densities. For nontangential dimers, however, periodic ordering of particles can result in better packing than aperiodic ordering, leading to a more complex competition

\footnotetext{
*k.milinkovic@uu.nl

${ }^{\dagger}$ m.dijkstra1@uu.nl
}

between packing and degeneracy entropy. The effects of this competition are important for understanding the phase behavior of these systems since synthesized dimers are often nontangential, e.g., because one sphere is "grown" onto another [4] or arising from techniques such as the lock and key [26].

Dumbbells, which consist of spheres with equal diameters and different separations, are a nontangential dimer particle system which has been widely studied using theoretical and simulation approaches [9-13]. It has been shown that these particles form aperiodic crystalline phases only for very large sphere separations and also that the underlying structure of the close packed crystalline phase does not change as the constituent sphere separation is reduced [10-12]. We note that two-dimensional aperiodic colloidal crystals have been experimentally observed in systems of dumbbell particles [27].

In this work, we address the question of how varying the sphere separation in systems of hard nontangential dimer particles with different constituent sphere diameters, which we will refer to as asymmetric dumbbells, affects the phase behavior. Hard-core systems are often used as a reference for systems with more complex interactions as a variety of colloidal and nanoparticle systems behave as nearly hard spheres [28,29]. Using Monte Carlo simulations and free energy calculations, we map out the phase diagram of systems of asymmetric dumbbells consisting of spheres of diameter ratio $d=0.5$. This $d$ value allows us to study different aspects of the phase behavior of dimer particles. The tangential particle phase behavior at this diameter ratio is relatively simple, with only isotropic and an aperiodic crystalline phase found to be stable [15]. We can therefore investigate whether reducing the sphere separation can have the same effect on the phase behavior as reducing the diameter ratio, i.e., stabilizing additional crystalline phases. We can also study the range of stability of aperiodic crystals in a system of nontangential dimers with a constituent sphere diameter ratio much lower than that of dumbbell particles.

The outline of this paper is as follows. In Sec. II, we briefly describe the simulation methods used and we present the calculated phase diagram in Sec. III A. In Secs. III B, IIIC, 
III D, and IIIE, we discuss the behavior and the properties of the various phases which we predict to be stable. Finally, in Sec. IV, we present our conclusions and also discuss what we can infer, based on our results, about the phase behavior of asymmetric dumbbell particles with other diameter ratios.

\section{METHOD}

\section{A. Model and simulation details}

We perform Monte Carlo (MC) simulations on systems of hard asymmetric dumbbell (AD) particles. These particles consist of two hard spheres with a diameter ratio $d=D_{S} / D_{L}$, where $D_{S}$ is the diameter of the smaller sphere and $D_{L}$ is the diameter of the larger sphere. We define the shape of an asymmetric dumbbell by the reduced sphere separation $L^{*}=$ $\left(2 L+D_{S}-D_{L}\right) / 2 D_{L}$, where $L$ is the distance between the centers of the constituent spheres and we have taken $D_{L}$ to be the unit of length (see Fig. 1). The quantity $L^{*}$ can be thought of as the length by which the smaller sphere is protruding from the larger one. In the limiting case of $L^{*}=D_{S} / D_{L}$, an asymmetric dumbbell particle reduces to a hard snowmanshaped particle, while for $L^{*}=0$ it becomes simply a hard sphere. The phase behavior in both of these limiting cases is known. In this work, we study systems of $\mathrm{AD}$ particles with constituent sphere diameter ratio $d=0.5$ and reduced sphere separations $L^{*}=0.1,0.2$, and 0.3 , while in the region of $0.3<L^{*}<0.5$, where the phase behavior becomes more intricate, we use a higher resolution.

In order to obtain the equations of state (EOS) for all particle shapes defined by $L^{*}$, we perform constant pressure Monte Carlo $(N P T)$ simulations on systems of $N \sim 500 \mathrm{AD}$ particles at pressure $P$ and temperature $T$. For all $L^{*}$ values studied, we obtain the isotropic fluid branches of the equations of state by increasing the pressure of a dilute isotropic fluid configuration in small steps. The solid branches of the equations of state are calculated in NPT MC expansion runs, where we start by generating a candidate crystal structure at high pressure and then we decrease the pressure slowly until the crystal melts. The crystal structures we consider as candidates are those which were found to be stable in Ref. [15] for hard snowman-shaped particles with a constituent sphere diameter ratio $d \leqslant 0.5$, namely, the structures based on the binary sphere $\mathrm{NaCl}$ and $\mathrm{CrB}$ crystals. In order to obtain the $\mathrm{AD}$ particle candidate crystal structures, we begin by generating the corresponding crystals of snowman-shaped particles in which the constituent spheres are tangential $\left(L^{*}=0.5\right)$, using the method described in Ref. [15]. We then sink the smaller sphere of each particle into the larger sphere in small steps, equilibrating the intermediate configurations, until we reach the desired $L^{*}$ value. We note that the positions of the large and small constituent spheres of an $\mathrm{AD}$ particle will deviate

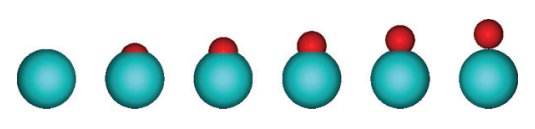

FIG. 1. (Color online) Asymmetric dumbbell particles with constituent sphere diameter ratio $d=D_{S} / D_{L}=0.5$ and reduced sphere separation $L^{*}=\left(2 L+D_{S}-D_{L}\right) / 2 D_{L}=0$ to $L^{*}=0.5$, in intervals of 0.1 .
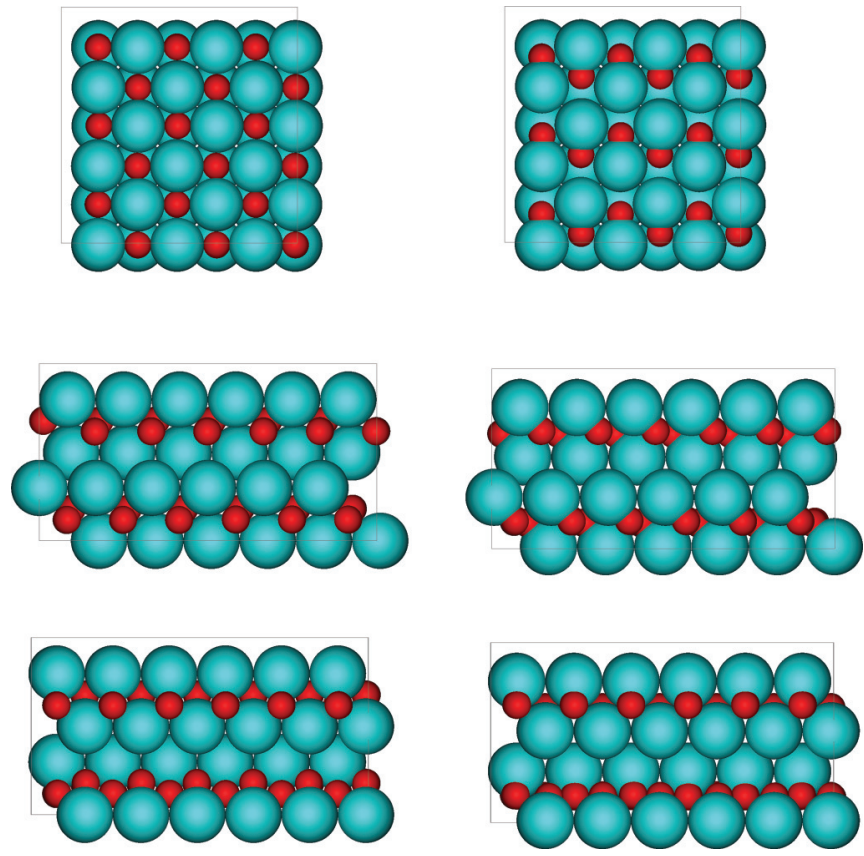

FIG. 2. (Color online) Example configurations of $\mathrm{NaCl}$ (top row) and $\mathrm{CrB}$ (middle and bottom rows, shown in two different planes) crystalline structures for snowman-shaped particles (lefthand column) and asymmetric dumbbell (AD) particles (right-hand column). Blue spheres represent the larger constituent spheres, red represent the smaller ones.

from the ideal lattice positions of the corresponding binary crystal. To illustrate this, we show examples of the $\mathrm{NaCl}$ - and CrB-based structures for both snowman-shaped particles and AD particles in Fig. 2.

As in the case of systems of dumbbell particles [12] and snowman-shaped particles [15], for AD particle systems it is possible to define three types of ordered structures. These are rotator phases (RP), periodic crystals, and aperiodic crystals. In a rotator phase (also referred to as a plastic crystal in the literature), the particle center of mass positions are on average located on a lattice but the particles can still rotate. Free rotation of a particle can be hindered by the surrounding particles, and at high densities correlations between the instantaneous particle positions and orientations can develop $[17,30]$. Periodic crystals have both periodic positional ordering of the particle centers of mass and periodic orientational ordering of the particles' major axes, and as such both the small and large constituent spheres are also periodically ordered even for nontangential AD particles. Finally, for aperiodic crystalline structures, the orientations of the particles' major axes are aperiodically ordered, leading to the centers of mass of the particles becoming disordered. We note that in the case of tangential $\mathrm{AD}$ particles (snowman-shaped particles) the constituent spheres in an aperiodic structure sit on a lattice, however, as $L^{*}$ is lowered, the sphere positions will deviate nonuniformly from the corresponding binary lattice sites.

The equations of state for the rotator and periodic crystal phases can be obtained from only a single set of expansion runs each since there is only one representative configuration of each for a given $L^{*}$ value. For aperiodic structures, however, there are multiple ways in which the particle orientations can 
be distributed. Hence, for each $L^{*}$ value, we perform expansion runs on a number of different aperiodic crystal structures and average the results to obtain the EOS of each candidate crystal. For tangential snowman-shaped particles, it was found that both the aperiodic and periodic structures of a candidate crystal have the same EOS [15]. However, we do not expect this to be the case for asymmetric dumbbell particle systems since orienting the particles periodically can, in principle, increase the overall density of an AD crystal at fixed pressure.

We finally comment on our choice of periodic configurations of the candidate crystals we use in this study. As the AD particle crystals are made from binary sphere crystals by joining touching large-small sphere pairs, there is clearly more than one way of obtaining periodic ordering. However, based on the results of previous studies of similar systems of dumbbell particles, we assume that, if chosen carefully, the specific periodic structure used will not affect the phase boundaries significantly. In Ref. [10] it was shown that three different types of periodically ordered structures in which the bonds of neighboring dumbbells are parallel have free energies that are very close to one another (with a difference of $\sim 0.01 k_{B} T$ per particle). Furthermore, in Ref. [13] it was shown that periodic orderings of dumbbells in which neighboring particle bonds were orthogonal to one another have a significantly higher free energy than the stable structure (up to $\sim 0.7 k_{B} T$ per particle). Hence, the periodic crystalline structures we study are formed with parallel neighboring bonds.

\section{B. Free energy calculations}

In order to determine which of the candidate crystalline phases are stable and to find the coexistence regions, we use free energy calculations. In this section, we outline the method we use and for a more detailed description we refer the interested reader to Ref. [15] in which hard snowman-shaped particles were considered.

We calculate the Helmholtz free energy $F$ of an isotropic fluid phase from the chemical potential which we obtain using the Widom particle insertion method [31]. In order to calculate the free energies of each of the candidate crystal structures, we use the thermodynamic integration method. This method involves integrating the free energy change along a reversible path which links the system of interest to a suitably chosen reference state for which the free energy is known. As the reference state we use a noninteracting Einstein crystal with the same underlying structure [32], and we obtain the lattice site positions and orientations for this crystal by averaging the center of mass positions and orientations of all particles using constant volume MC simulations.

To link the system of interest to the reference crystal, we tether the particle positions to the corresponding lattice sites using harmonic springs, and their orientations using a binding potential. We vary the strength of the tethering such that in one limiting case the particles are completely fixed to their lattice sites, while in the other they can move freely. Note that for rotator phases, only the particle center of mass tethering is required. In order to complete the transformation from the crystal of interest to the noninteracting Einstein crystal, we use a soft potential which allows the particle interactions to be tuned between the hard-core and the interaction free limit. The resulting integration path linking the system of interest to the reference crystal is as follows: The springs are turned on in stages until the particles are fixed to the lattice sites of the reference crystal, then the softness of the particles is gradually increased, through the soft potential, until the system reduces to a noninteracting Einstein crystal. Integrating over this path gives us the free energy at a single state point. In order to account for finite size effects, we calculate the free energy for various system sizes $N$ at this state point and extrapolate the results to the thermodynamic limit [33]. Although the original extrapolation method was designed for hard spheres [33], it was shown in Ref. [13] that it also works well for systems consisting of hard dumbbells. Finally, to obtain the free energy as a function of density, we integrate the free energy change over the EOS of the structure of interest.

For aperiodic crystalline structures, an additional degeneracy entropy contribution to the free energy has to be taken into account since the different aperiodic realizations have the same free energy. The degeneracy $\Omega$ of a particular crystal structure is defined as the number of possible configurations of particle orientations, and for crystalline structures of $\mathrm{AD}$ particles the degeneracy will be the same as for the snowman-shaped particle crystals that they are based on. For the candidate crystalline structures considered here, the degeneracy has been calculated in Ref. [15] by following the series expansion method given in Ref. [35]. The zeroth order term, which is equivalent to the Bethe approximation, depends solely on the number of large-small sphere nearest neighbors (defined as $q$ ), while the higher order terms are calculated specifically for each lattice. While both the $\mathrm{NaCl}$ and $\mathrm{CrB}$ lattices have the same $q$ value, it was found in Ref. [15] that the higher order terms differ and hence their degeneracies also differ slightly. For the aperiodic $\mathrm{NaCl}$ phase, the degeneracy is given by $\ln \Omega / N=0.8945$ and for the aperiodic $\mathrm{CrB}$ phase it is given by $\ln \Omega / N=0.8933$. Having calculated the equations of state and the free energies, we determine bulk coexistence densities by equating the pressure and the chemical potential of the two coexisting phases.

\section{RESULTS}

\section{A. Phase diagram}

Using the methods described in Sec. II B, we have calculated the free energies of isotropic fluid and candidate crystalline phases of asymmetric dumbbell particle systems for a range of reduced AD constituent sphere separations $L^{*}$. Based on the obtained free energies, we have determined the stable phases, calculated the coexistence densities, and, finally, we have constructed the phase diagram shown in Fig. 3.

For $L^{*}=0$ we recover the phase behavior of pure hard spheres, with stable isotropic fluid and FCC phases. As we go towards higher values of $L^{*}$, in the range of $0<L^{*} \lesssim 0.207$, the asymmetric dumbbells form only stable isotropic fluid and FCC rotator phases. These rotator phases form spontaneously in MC compression runs and they are characterized by the centers of mass of the particles being located, on average, on FCC crystal lattice sites. At high densities, in order to optimize the packing, this organization changes to the large spheres of 


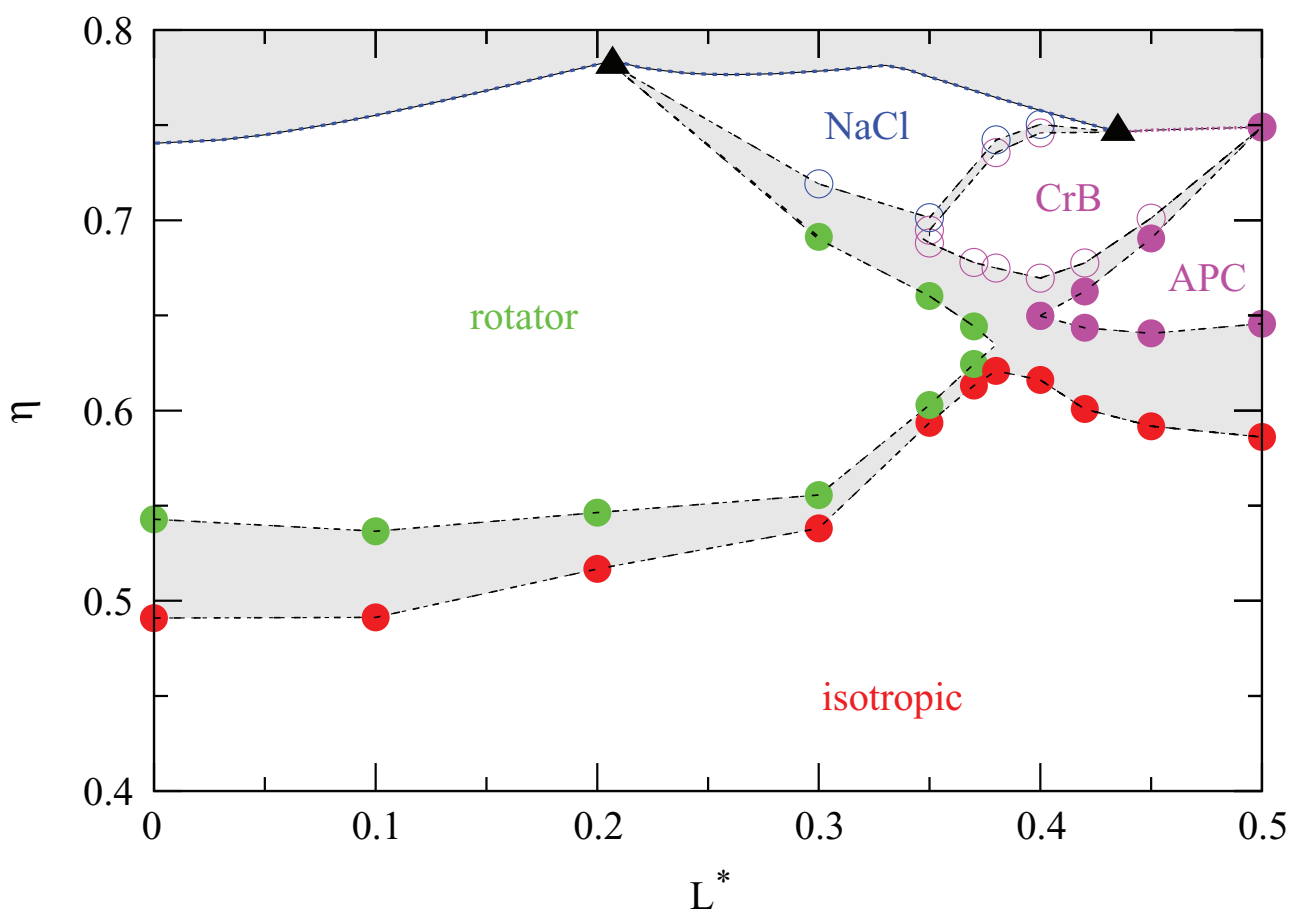

FIG. 3. (Color online) Phase diagram of hard asymmetric dumbbell (AD) particles with sphere diameter ratio $d=D_{S} / D_{L}=0.5$ in the $L^{*}-\eta$ representation, with $L^{*}=\left(2 L+D_{S}-D_{L}\right) / 2 D_{L}$ ranging from 0 (the hard sphere) to $L^{*}=0.5$ (the tangential snowman-shaped particle) and $\eta=v_{0} N / V$, where $v_{0}$ is the volume of an $\mathrm{AD}$ particle for a given $L^{*}$ value and $V$ is the total volume of the system. APC refers to the aperiodic $\mathrm{CrB}$ crystal structure, $\mathrm{CrB}$ denotes the periodic $\mathrm{CrB}$ crystalline phase, and $\mathrm{NaCl}$ denotes the periodic $\mathrm{NaCl}$ crystalline phase. Circles indicate coexisting phases, while the lines are intended to guide the eye. At the top of the plot, we indicate the density of closest packing, with triangles indicating crossover points from one close packed structure to another. Coexistence densities for $L^{*}=0$ are taken from Ref. [34] and for $L^{*}=0.5$ they are taken from Ref. [15].

the AD particles (instead of the centers of mass of the particles) occupying the FCC lattice sites, with the small spheres still moving within the free space. As we approach close packing, the ADs no longer rotate but become frozen in place.

Moving towards still higher $L^{*}$ values, with $L^{*}$ in-between 0.207 and $\sim 0.35$, the phase behavior complicates further, with orientationally periodic $\mathrm{NaCl}$ phases found to be stable at high densities. In this $L^{*}$ region, we expect to find stable isotropic fluid, $\mathrm{RP}$, and periodic $\mathrm{NaCl}$ phase with increasing density. Moreover, we can see from the phase diagram that, as $L^{*}$ is increased within this region, the periodic $\mathrm{NaCl}$ phase becomes increasingly more stable with respect to the rotator phase, as does the isotropic fluid phase. At $L^{*}=0.35$, an additional structure, a periodic CrB crystal, emerges as stable. Hence, the expected phase behavior from low to high densities for the system characterized by $L^{*}=0.35$ is as follows: isotropic fluid, an FCC rotator phase followed by a periodic $\mathrm{CrB}$, and finally a periodic $\mathrm{NaCl}$. For $0.35<L^{*} \lesssim 0.38$, the range of stability of the periodic $\mathrm{CrB}$ phase increases, while the density ranges in which the rotator and now also periodic $\mathrm{NaCl}$ phases are stable decrease. Finally, at $L^{*} \sim 0.38$, the FCC rotator phase vanishes completely and we predict a direct isotropic fluid-periodic $\mathrm{CrB}$ phase transition. At high densities, a periodic $\mathrm{NaCl}$ is still found to be stable, although we note that the structure has become somewhat modified. This will be discussed further in Sec. III D.

Slightly increasing the value of $L^{*}$ even further, to $L^{*} \sim$ 0.4 , results in the emergence of a stable orientationally aperiodic $\mathrm{CrB}$ phase, which we will refer to from now on as APC. The range of stability of this phase grows all the way up to $L^{*}=0.5$ at the expense of both the periodic $\mathrm{CrB}$ and the isotropic fluid phase. For $L^{*}>0.435$, we find that the periodic $\mathrm{NaCl}$ phase is no longer stable; this is also where the CrB crystal becomes the best packed structure. Finally, at the snowmanshaped particle limit, corresponding to $L^{*}=0.5$, only stable isotropic fluid and aperiodic $\mathrm{CrB}$ phases are found [15].

\section{B. Stability range of rotator phases}

A large portion of the phase diagram in this study is dominated by rotator phases: they are found to be stable in the range of $0<L^{*} \lesssim 0.38$. Such a large range of rotator phase stability was also found in the similar dimer systems of snowman-shaped particles (SM) [15] and dumbbells (DB) $[10,11,36]$. In this section, we turn our attention to the rotator phase behavior in these systems.

We discuss the phase behavior of the rotator phases in terms of two parameters: the end-to-end length $x$ of a particle and the single particle volume $v_{0}$. For the AD particles studied here, the end-to-end length is given by $x=D_{L}\left(1+L^{*}\right)$, for SM particles it is given as $x=D_{L}(1+d)$, while for DB particles it is given by $x=D_{L}\left(1+L / D_{L}\right)$ where $L$ is the distance between the centers of the constituent spheres. In Fig. 4, we illustrate these particles for several $x$ values.

We first note that the particle end-to-end length at which an isotropic fluid-rotator phase transition is no longer observed, 


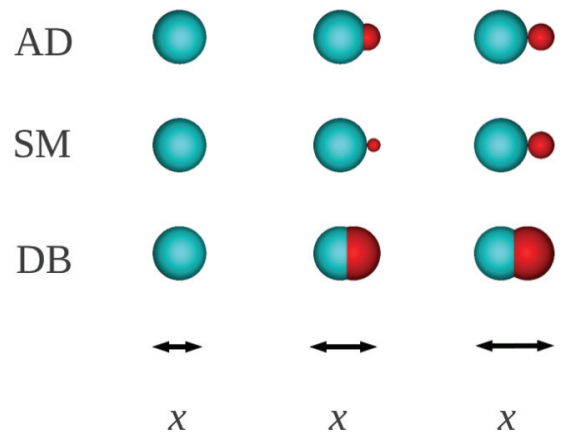

FIG. 4. (Color online) Top to bottom: asymmetric dumbbell (AD), snowman-shaped (SM), and dumbbell (DB) particles with end-to-end lengths (from left to right) $x / D_{L}=1,1.25$, and 1.5.

and at which a direct isotropic fluid-solid transition is found, is similar for all of these systems. This can be seen in Fig. 5(a), where we show, for the three systems, the phase diagram of the isotropic fluid-rotator and rotator-solid phase transitions as a function of the particle end-to-end length. Of the three, the systems of snowman-shaped particles form rotator phases which are stable for a slightly larger particle end-to-end length than in the case of the other two systems, both of which are remarkably close. We also see that the packing fractions at which isotropic fluid-rotator phase coexistence is predicted are lowest for snowman-shaped particles (for a given end-to-end length), followed by the $\mathrm{DB}$ and $\mathrm{AD}$ particles, while the
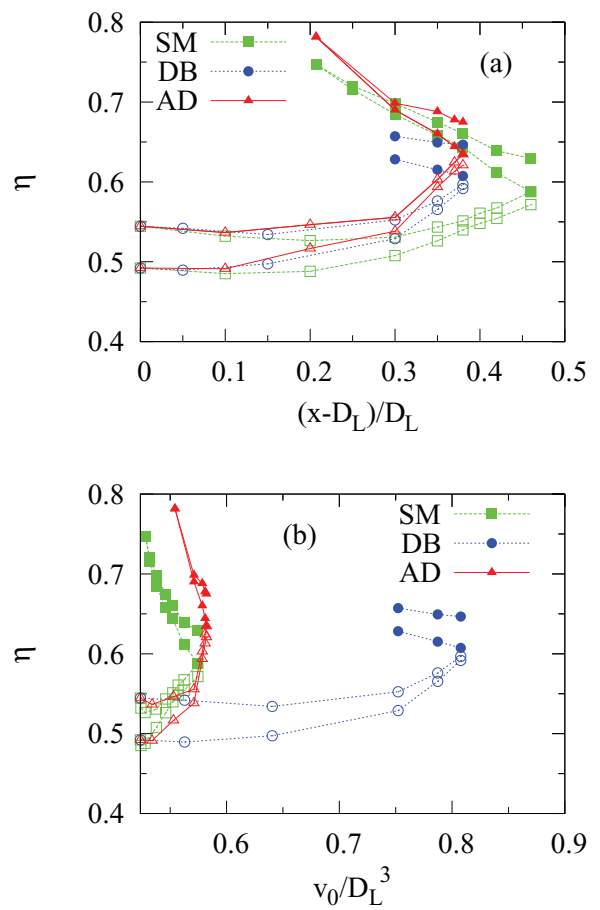

FIG. 5. (Color online) Phase diagram showing the isotropic fluid-rotator (open symbols) and rotator-solid (filled symbols) phase transitions in systems of hard dumbbell (DB), hard snowman-shaped (SM), and hard asymmetric dumbbell (AD) particles, as a function of (a) reduced end-to-end length of a particle $\left(x-D_{L}\right) / D_{L}$ and (b) particle volume $v_{0} / D_{L}^{3}$. $\eta$ denotes the packing fraction; $D_{L}$ is the unit of length. Data for DB particles are taken from Ref. [36] and for SM particles from Ref. [15]. packing fractions at which rotator-solid phase coexistence is predicted are lowest for the DB particles, followed by the $\mathrm{SM}$ and $\mathrm{AD}$ particles. Consequently, the snowman-shaped particles have the largest packing fraction range for which the rotator phases are stable. This indicates that snowmanshaped particles, which are the only particles consisting of tangential spheres, favor the rotator phase significantly more than the other two systems, in which the constituent spheres are overlapping. The $\mathrm{AD}$ particles studied here have an intermediate sized region of stability, while the dumbbells have the smallest. These observations can be understood in terms of the geometry of the different particle shapes. For a given end-to-end length, the dumbbell has the largest volume, which will clearly lead to more interactions between particles at high packing fractions, making free rotation unfavorable. Conversely, the snowman-shaped particles, which have the largest range of the rotator phase stability, are the ones with the smallest volume. In Fig. 5(b), we show the isotropic fluidrotator and rotator-crystal phase transitions as in Fig. 5(a), but now as a function of the volume of a single particle $v_{0}$ instead of its end-to-end length $x$. From this, we can clearly see that the volume of dumbbell particles forming rotator phases is much larger than the volumes of the dimer particles in the rotator phases of the other two systems.

In conclusion, we find that the key factor in determining when the rotator phase of a dimer particle system stops being stable is the particle end-to-end length, while the particle volume determines the packing fraction range of stability.

\section{Orientational reorganization}

The presence of aperiodic and rotator phases in the phase diagram of asymmetric dumbbell particles is a direct consequence of the $\mathrm{AD}$ particles having orientational degrees of freedom. Both of these types of phases are characterized by a degree of orientational disorder which results in an entropy gain that stabilizes them. As discussed previously, for $L^{*}$ values below $\sim 0.207$, the only stable ordered structures are the rotator phases, while above this $L^{*}$ value the stability range of the rotator phases shifts to mid-densities and the $\mathrm{NaCl}$ phase emerges as the stable structure at high densities. Thus, we see from the phase diagram that as $L^{*}$ is increased, the stability range of the rotator phases decreases.

In the case of aperiodic structures, the scenario is reversed. They are stable only for large $L^{*}$ values and their range of stability grows with $L^{*}$, until finally at $L^{*}=0.5$ the aperiodic structures completely replace the other solids. In these systems, the particles are localized both positionally and orientationally, although the particle orientations are not arranged in any particular way (they form a disordered set) and this is where the gain in entropy, compared to a periodic structure, comes from.

Our aim in this section is to investigate the orientational behavior of the AD particles in rotator and aperiodic phases. To do this, we calculate the first and second order orientational correlators for a single, randomly chosen particle, over a long $\mathrm{MC}$ simulation run. These time correlation functions are given as

$$
\begin{gathered}
P_{1}(t)=\langle\cos \theta(t)\rangle, \\
P_{2}(t)=\frac{1}{2}\left\langle 3 \cos ^{2} \theta(t)-1\right\rangle,
\end{gathered}
$$


where $\theta(t)$ is the angle between the initial orientation of the particle and its orientation at time $t$ (given in units of MC cycles) and $\langle\ldots\rangle$ denotes the ensemble average. $P_{1}(t)$ takes values in the range of -1 to 1 , where 1 corresponds to perfect alignment and -1 corresponds to a head-to-tail arrangement. $P_{2}(t)$ takes values from -0.5 to 1 , where 1 again corresponds to perfect alignment and -0.5 indicates an orthogonal arrangement. Generally, we expect both correlators to have a high value for any solid phase and to decay to 0 very quickly for isotropic fluid phases, and we expect the attained values in both of these cases not to change in time. We note here that the rotational motion of nontangential dimers has previously been studied in dilute suspensions using combined experimental and theoretical techniques $[37,38]$.

We plot $P_{2}(t)$ for rotator phases with $L^{*}=0.1,0.3$, and 0.35 in Fig. 6(a). The simulated systems are in all cases at a packing fraction of $\eta \sim 0.64$ which is well inside the stable rotator phase regime. For $L^{*}=0.1$, we see that the correlation function decays rapidly to 0 , indicating that the particles are rotating freely. For the intermediate value of $L^{*}=0.3$, we see an initial drop in $P_{2}(t)$ to below 0 , followed by fluctuations around 0 until the correlation function finally settles to a value close to 0 . The negative value of $P_{2}(t)$ indicates that the particle is aligned roughly orthogonal to its initial orientation. As the centers of mass of the AD particles are positioned on average on an FCC lattice, and as for $L^{*}=0.3$ only one small AD constituent sphere can fit in each of the gaps in-between the larger spheres, there are six directions along which an $\mathrm{AD}$ particle is on average mostly oriented. Each of these six directions is orthogonal to the neighboring ones, hence, when a particle reorients its new orientation is most likely to be perpendicular to the previous one. The decay to zero of the correlation function at longer times indicates that the particle does not favor any of these directions in particular. For $L^{*}=0.35$, the effect is more pronounced. The jagged decay of the $P_{2}(t)$ line towards 0 indicates that a particle keeps its orientation for a longer time before reorienting again. Further confirmation of this can be seen in the inset of Fig. 6(a), where we show the instantaneous value of $\left[3 \cos ^{2} \theta(t)-1\right] / 2$. As we can see, the values this function takes are mostly close to -0.5 and 1 , which correspond to perpendicular orientations of the particle. Clearly, the distribution of orientations of a single particle in a rotator phase become increasingly nonuniform, i.e., rotation becomes more hindered with increasing $L^{*}$, until the rotator phase finally becomes unstable at $L^{*} \sim 0.38$.

In Fig. 6(b), we plot $P_{1}(t)$ for a system of $\mathrm{AD}$ particles with $L^{*}=0.45$, which forms a stable aperiodic $\mathrm{CrB}$ crystal at intermediate densities and a periodic $\mathrm{CrB}$ crystal at high densities. The periodic structure is stable above $\eta=0.701$, the aperiodic structure is stable in the region of $\eta=0.641$ to 0.690 , while the isotropic fluid phase is stable below $\eta=0.592$. The $P_{1}(t)$ curves plotted here correspond to four different densities: a very high density where the stable phase is a periodic $\mathrm{CrB}$ (at $\eta=0.714$ ), a lower density where we predict a stable aperiodic $\mathrm{CrB}$ (at $\eta=0.648$ ), a density within the aperiodic $\mathrm{CrB}$-isotropic fluid phase coexistence region (at $\eta=0.611$ ), and a density where the system is an isotropic fluid (at $\eta=0.501$ ). The correlation function for the system in the isotropic fluid phase decays rapidly to $P_{1}(t) \sim 0$ as expected. In the periodic and aperiodic crystal structures, the
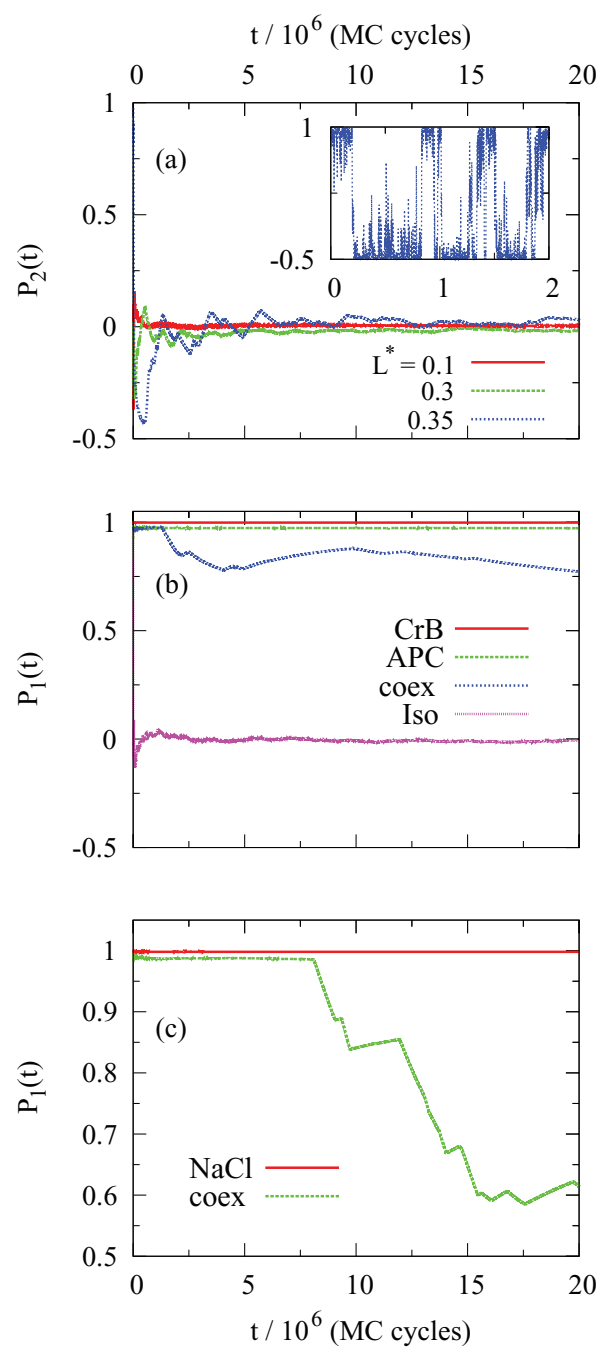

FIG. 6. (Color online) (a) Second order orientational correlation function $P_{2}(t)$ plotted as a function of simulated time $t$ for asymmetric dumbbell $(\mathrm{AD})$ particle systems with $L^{*}=0.1,0.3$, and 0.35 , at packing fraction $\eta \sim 0.64$, at which the systems form stable rotator phases. Inset: [ $\left.3 \cos ^{2} \theta(t)-1\right] / 2$ as a function of simulated time for the system with $L^{*}=0.35$. (b) First order orientational correlator $P_{1}(t)$ as a function of simulated time for a system with $L^{*}=0.45$ at four densities: $\mathrm{CrB}$ here indicates periodic $\mathrm{CrB}$ phase, $\mathrm{APC}$ indicates aperiodic $\mathrm{CrB}$ phase, coex denotes the system in the APC-isotropic fluid phase coexistence region, and Iso indicates isotropic fluid phase. (c) $P_{1}(t)$ for a system with $L^{*}=0.3$ at two densities: $\mathrm{NaCl}$ indicates periodic $\mathrm{NaCl}$ phase at $\eta=0.74$, and coex denotes the system in the $\mathrm{NaCl}$-rotator phase coexistence region at $\eta=0.685$.

orientations of particles are fixed and the correlation functions decay to a constant, high value. This value is slightly lower for the aperiodic structure than for the periodic structure since the $\mathrm{APC}$ is at a lower density, which leaves more free space for fluctuations in the positions and orientations of the particles.

For the system within the aperiodic $\mathrm{CrB}$-isotropic fluid phase coexistence region, we see a slow reorganization of particles, as the $P_{1}(t)$ value does not remain constant but instead decays gradually. This metastable structure does not, however, melt completely into the isotropic fluid phase, as the particle orientations remain correlated. Once the orientations of particles within an aperiodic crystal structure are no longer 


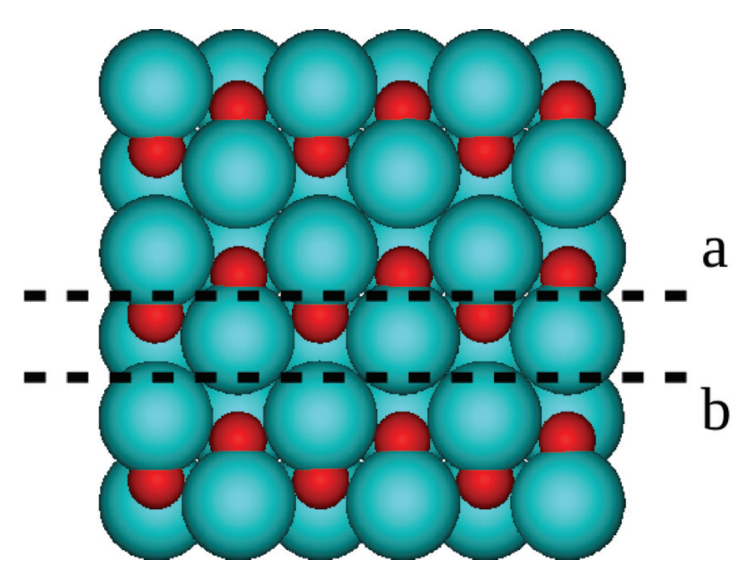

FIG. 7. (Color online) Example configuration of the modified $\mathrm{NaCl}$ crystalline structure of asymmetric dumbbell (AD) particles with $L^{*}=0.3$. Blue spheres represent the larger constituent spheres, red represent the smaller ones. Large constituent spheres along the dashed line denoted by $a$ are slightly separated, while those along the dashed line denoted by $b$ are touching.

fixed, the crystal can no longer be considered as aperiodic and hence it loses the degeneracy entropy contribution to the free energy. We also see a similar process of orientational reorganization of particles for a system with $L^{*}=0.3$ within the periodic $\mathrm{NaCl}$-rotator phase coexistence region, as shown in Fig. 6(c). We note that we only see orientational reorganization of the particles within crystals in the density regions where we have predicted them to be unstable.

\section{Modified NaCl structure}

We now turn our attention to the modified $\mathrm{NaCl}$ crystal structures found to be stable at very high densities in the reduced sphere separation range of $0.207 \lesssim L^{*} \lesssim 0.435$, as mentioned in Sec. III A. The periodic crystal structures we consider are those in which neighboring $\mathrm{AD}$ particles are parallel with constituent sphere bonds oriented at $180^{\circ}$ to one another since we find that these structures can pack better than those with parallel bonds oriented at $0^{\circ}$. In an unmodified $\mathrm{NaCl}$ structure, each large constituent sphere has 12 large sphere nearest neighbors at high density (4 in each plane). However, in the range of $0.207 \lesssim L^{*} \lesssim 0.435$, we find that the $\mathrm{NaCl}$ structure can, at high pressure, achieve better packing by modifying such that each large sphere now has 6 large sphere nearest neighbors ( 2 in each plane). This is illustrated in Fig. 7. Here, the large spheres along the line denoted by $a$ become slightly separated, to fit the small spheres better, while those along the line denoted by $b$ remain touching. The transformation from unmodified to modified $\mathrm{NaCl}$ when compressing a system, as well as from modified to unmodified when expanding, does not result in a noticeable effect on the equation of state. We note that a similar modification was observed in systems of snowman-shaped particles with diameter ratios $d>0.414$ [15].

To elucidate what the role of this modification is in the stability of the $\mathrm{NaCl}$ phase, we calculate the free energy of both the modified and the unmodified $\mathrm{NaCl}$ structures at a range of densities. We obtain the unmodified structure at the desired density by simply generating an $\mathrm{NaCl}$ configuration at this
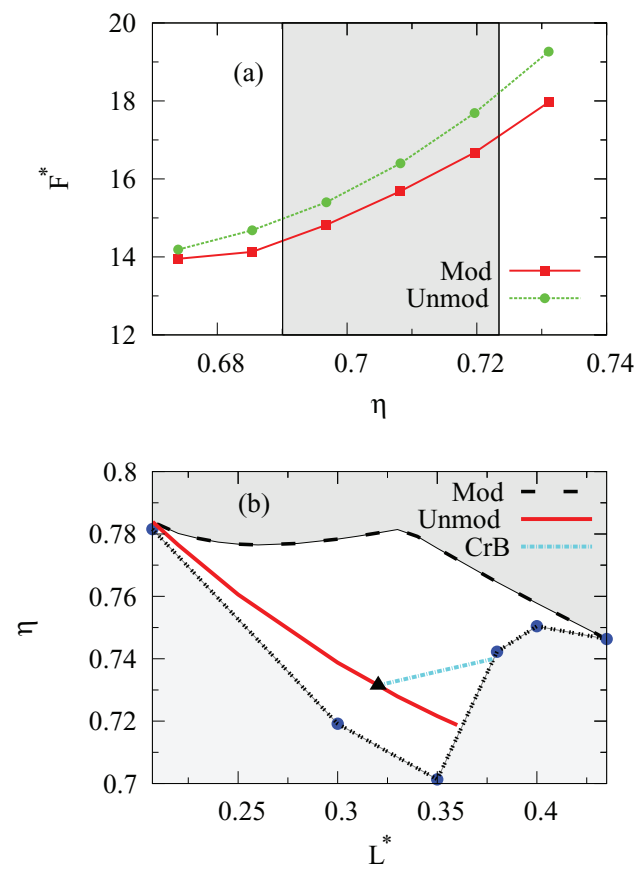

FIG. 8. (Color online) (a) Free energy per particle $F^{*}=\beta F / N$ as a function of packing fraction $\eta$ for both modified (red squares) and unmodified (green circles) $\mathrm{NaCl}$ structures for a system of asymmetric dumbbell (AD) particles with $L^{*}=0.3$. Shaded region indicates the predicted rotator-modified $\mathrm{NaCl}$ coexistence region. (b) Density at close packing of the modified (long-dashed black line) and the unmodified (solid red line) periodic $\mathrm{AD} \mathrm{NaCl}$ structures as a function of $L^{*}$. Dashed-dotted line with circles indicates the $\mathrm{NaCl}$ melting line. Unshaded region represents the range of predicted $\mathrm{NaCl}$ stability. We also include the line of close packing for the periodic $\mathrm{CrB}$ structure (short-dashed light blue line). Black triangle indicates the $L^{*}$ value at which the $\mathrm{CrB}$ phase becomes better packed than the unmodified $\mathrm{NaCl}$ structure.

density, while to obtain the modified structure we uniformly expand an equilibrated close packed modified configuration to the same density. We then calculate the free energies at each of these state points, and show the results for a system with $L^{*}=0.3$ in Fig. 8(a).

What is immediately apparent is that in the density region for which the $\mathrm{NaCl}$ phase was found to be stable, the free energy of the unmodified structure for $L^{*}=0.3$ is significantly higher than that of the modified structure. At lower densities, the free energies become closer, but only below the coexistence region. For $L^{*}=0.35$, we also find that the free energy of the modified $\mathrm{NaCl}$ structure is lower than that of the unmodified $\mathrm{NaCl}$ in the region of stability. This implies that the stability of the periodic $\mathrm{AD} \mathrm{NaCl}$ phase is significantly enhanced by this modification, as a higher free energy (corresponding to the unmodified $\mathrm{NaCl}$ structure) would result in the coexistence regions being predicted at higher densities.

Furthermore, when we consider the best packings that the unmodified $\mathrm{NaCl}$ structures can achieve in the context of the predicted stability range of the $\mathrm{NaCl}$ phase, we see that these are only slightly higher than the packings at which the $\mathrm{NaCl}$ phases first become stable. This is shown in Fig. 8(b). Additionally, it can be seen that the $L^{*}$ value at which the periodic $\mathrm{CrB}$ phase becomes better packed than the $\mathrm{NaCl}$ phase 
is also lower for the unmodified $\mathrm{NaCl}$ structure. Hence, while we would still expect the $\mathrm{NaCl}$ phase to be present in the phase diagram in the absence of the modification, its range of stability would be significantly smaller.

\section{E. Destabilizing aperiodic structures}

In contrast to the phase behavior predicted for tangential snowman-shaped particles [15], where all stable crystal structures are orientationally aperiodic, for AD particle systems aperiodic structures have only a very small range of stability. A tangential particle system at high density can be thought of as an equimolar binary sphere mixture with certain pairs of touching spheres connected, therefore the constituent spheres in a crystal of snowman-shaped particles will have the same positions in both periodic and aperiodic realizations. What is different is that there are many more ways of constructing the aperiodic crystals, which results in these having a higher entropy and with that a lower free energy. However, as we lower $L^{*}$ below 0.5 (the snowman-shaped particle limit), the constituent sphere positions in the resulting crystals will diverge more and more from their positions on the corresponding ideal binary lattice. For periodic crystals, this distortion of the lattice will be uniform, while for aperiodic
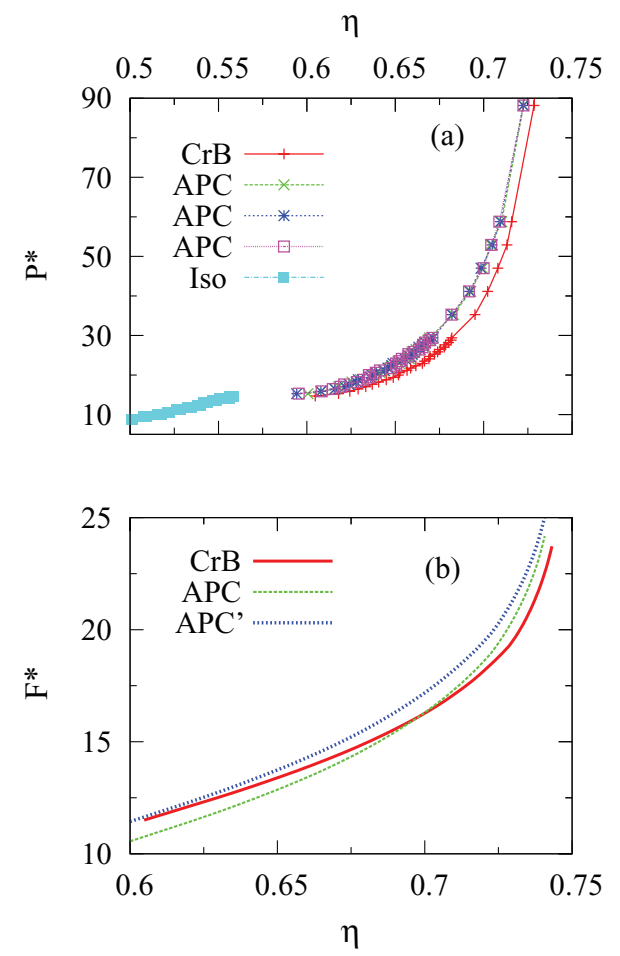

FIG. 9. (Color online) (a) Equations of state for the periodic and three aperiodic structures for the $\mathrm{AD} \mathrm{CrB}$-based crystal with $L^{*}=$ 0.45. $P^{*}=\beta P D_{L}^{3}$ denotes the reduced pressure and $\eta=\rho v_{0}$ is the packing fraction, where $v_{0}$ is the volume of an AD particle. (b) Free energy per particle $F^{*}=\beta F / N$ as a function of packing fraction $\eta$ for periodic and aperiodic structures of the CrB-based crystal with $L^{*}=0.45$. $\mathrm{CrB}$ denotes the free energy of the periodic and APC the free energy of the aperiodic phase. APC ${ }^{\prime}$ denotes the free energy of the aperiodic phase without the degeneracy entropy term: $F^{*}=$ $(\beta F+\ln \Omega) / N$. structures it will be nonuniform and, as a consequence, the way the particles are oriented will influence the packing.

As an illustration of this behavior, in Fig. 9(a), we plot the equations of state for both aperiodic and periodic $\mathrm{CrB}$ AD particle crystals with $L^{*}=0.45$. As we can see, the EOS curves of the three aperiodic structures lie on top of each other (within statistical error), while the EOS of the periodic structure shows higher packing for a given pressure at all densities. From the phase diagram (Fig. 3) we see that, for the system with $L^{*}=0.45$, the APC structure is stable at densities in the range of $0.65<\eta<0.69$, even though it is less well packed than the periodic structure in this density range. This indicates that the aperiodic structure in this region is stabilized by degeneracy, i.e., the entropic gain associated with the aperiodicity of the particle orientations outweighs the loss in packing. The importance of the degeneracy can also be seen in Fig. 9(b), where we show that the free energy per particle is lower at all densities in the periodic $\mathrm{CrB}$ phase than in the APC phase if the degeneracy entropy term is removed, while if it is included, the free energy of the aperiodic phase is lower up to $\eta \sim 0.7$.

\section{CONCLUSIONS}

We have investigated the phase behavior of systems of hard asymmetric dumbbell particles with a fixed constituent sphere diameter ratio of $d=0.5$, using Monte Carlo simulations and free energy calculations. The particle shapes studied here range from the snowman-shaped particle to the hard sphere. At the snowman-shaped particle limit, only isotropic fluid and aperiodic $\mathrm{CrB}$ phases were predicted to be stable. Reducing the separation of the constituent spheres of the AD particles results in the phase behavior becoming more complex, as we now find stable isotropic fluid, $\mathrm{FCC}$ rotator, periodic $\mathrm{NaCl}$ and both periodic and aperiodic $\mathrm{CrB}$ phases.

For low sphere separations, we predict FCC rotator phases to be stable for a large region of the phase diagram. We compare this region to the ranges of stability of the rotator phases in systems consisting of hard snowman-shaped and dumbbell particles, as these three shapes belong to the same class of hard-sphere dimer particles. We find that the particle end-to-end length at which the rotator phases are no longer present in the phase diagram is similar in all cases. This indicates that the end-to-end length is more important for destabilizing the rotator phases of dimer particles than the individual particle volumes since these vary greatly between the three systems. We also see that, as the sphere separation of the $\mathrm{AD}$ particles is increased, free rotation of the particles in a rotator phase becomes increasingly hindered by the surrounding particles until the rotator phase finally becomes destabilized.

For intermediate values of the sphere separation, we predict a periodic $\mathrm{NaCl}$ phase to be stable at high densities. However, the observed crystal structure is not the standard, but instead a slightly modified $\mathrm{NaCl}$ crystal, and we find that the origin of this modification lies in the tendency of the system to optimize its packing, i.e., in this way the $\mathrm{NaCl}$ phase achieves better packing at very high pressures. The modification extends significantly the range of stability of the $\mathrm{NaCl}$ phase. We confirm this by calculating and comparing the free energies 
of the modified and unmodified $\mathrm{NaCl}$ structures, and also by comparing the best possible packing of the unmodified $\mathrm{NaCl}$ to that of the modified $\mathrm{NaCl}$ and $\mathrm{CrB}$ structures.

At high values of the asymmetric dumbbell constituent sphere separation, as we approach the snowman-shaped particle limit, we find a region in which aperiodic phases are stable. We note that the range of stability of the aperiodic crystals of asymmetric dumbbells is significantly smaller than in the case of snowman-shaped particles, where all predicted crystalline phases are orientationally aperiodic. This suppression of aperiodicity in systems of asymmetric dumbbells (as compared to the phase behavior of snowman-shaped particles) is due to the positions of the constituent spheres deviating from the crystal lattice sites as the sphere separation is reduced. The density is no longer invariant to the orientation of the particles and the degeneracy entropy is not always sufficiently large to overcome the free energy gain arising from the better packing of periodic structures.

Finally, we discuss what we can infer from our results about the general phase behavior of asymmetric dumbbell particles. Based on this work and previous studies of snowman-shaped and dumbbell particles, we would always expect a rotator phase to be present in the phase diagram of systems of dimers with small diameter ratios and/or sphere separations. We would also expect that reducing the sphere separation, compared to tangential particles with the same sphere diameter ratio, will at first destabilize the aperiodic structure, as the periodic ordering of particles will give better packing. This will not be the case for $d<0.414$, as the $\mathrm{NaCl}$ structure predicted to be stable for tangential dimers in this $d$ range will have the same packing for periodic and aperiodic structures as the separation is reduced. It is also possible that as the sphere separation is reduced, the best packed crystalline phase will change relative to that of the corresponding tangential particle system. This can lead to the appearance of an additional stable phase at high densities, although we note that for dumbbell particles no additional structures were found to be stable, indicating that this will be the case only for certain sphere diameter ratios.

\section{ACKNOWLEDGMENTS}

This work was financed by an NWO-VICI grant and is part of the research program of the Stichting door Fundamenteel Onderzoek der Materie (FOM) which is financially supported by the Nederlandse organisatie voor Wetenschappelijk Onderzoek (NWO).
[1] A. M. Wierenga, T. A. J. Lenstra, and A. P. Philipse, Colloids Surf., A 134, 359 (1998).

[2] A. Kuijk, A. van Blaaderen, and A. Imhof, J. Am. Chem. Soc. 133, 2346 (2011).

[3] J.-M. Meijer, F. Hagemans, L. Rossi, D. V. Byelov, S. I. R. Castillo, A. Snigirev, I. Snigireva, A. P. Philipse, and A. V. Petukhov, Langmuir 28, 7631 (2012).

[4] A. Perro, S. Reculusa, E. Bourgeat-Lami, E. Duguet, and S. Ravaine, Colloids Surf., A 284, 78 (2006).

[5] C. J. DeSantis and S. E. Skrabalak, Langmuir 28, 9055 (2012).

[6] K. Wojciechowski, Phys. Lett. A 122, 377 (1987).

[7] J. D. McCoy, S. Singer, and D. Chandler, J. Chem. Phys. 87, 4853 (1988).

[8] S. J. Smithline, S. W. Rick, and A. D. J. Haymet, J. Chem. Phys. 88, 2004 (1988).

[9] K. W. Wojciechowski, D. Frenkel, and A. C. Branka, Phys. Rev. Lett. 66, 3168 (1991).

[10] C. Vega, E. P. A. Paras, and P. A. Monson, J. Chem. Phys. 96, 9060 (1992).

[11] C. Vega, E. P. A. Paras, and P. A. Monson, J. Chem. Phys. 97, 8543 (1992).

[12] M. Marechal and M. Dijkstra, Phys. Rev. E 77, 061405 (2008).

[13] M. Kowalik and K. W. Wojciechowski, J. Non-Cryst. Solids 354, 4354 (2008).

[14] A. Šarić, B. Bozorgui, and A. Cacciuto, J. Phys. Chem. B 115, 7182 (2011).

[15] M. Dennison, K. Milinković, and M. Dijkstra, J. Chem. Phys. 137, 044507 (2012)

[16] J. D. Forster, J.-G. Park, M. Mittal, H. Noh, C. F. Schreck, C. S. O'Hern, H. Cao, E. M. Furst, and E. R. Dufresne, ACS Nano 5, 6695 (2011).
[17] I. D. Hosein, S. H. Lee, and C. M. Liddell, Adv. Funct. Mater. 20, 3085 (2010).

[18] M. Okubo, Z. Wang, E. Ise, and H. Minami, Colloid Polym. Sci. 279, 976 (2001).

[19] S. Reculusa, C. Poncet-Legrand, A. Perro, E. Duguet, E. Bourgeat-Lami, C. Mingotaud, and S. Ravaine, Chem. Mater. 17, 3338 (2005).

[20] D. Nagao, M. Hashimoto, K. Hayasaka, and M. Konno, Macromol. Rapid Commun. 29, 1484 (2008).

[21] D. J. Kraft, J. Groenewold, and W. K. Kegel, Soft Matter 5, 3823 (2009).

[22] D. Nagao, C. M. van Kats, K. Hayasaka, M. Sugimoto, M. Konno, A. Imhof, and A. van Blaaderen, Langmuir 26, 5208 (2010).

[23] E. B. Mock and C. F. Zukoski, Langmuir 26, 13747 (2010).

[24] N. Chaturvedi, B. Juluri, Q. Hao, T. Huang, and D. Velegol, J. Colloid Interface Sci. 371, 28 (2012).

[25] M. Hoffmann, M. Siebenburger, L. Harnau, M. Hund, C. Hanske, Y. Lu, C. S. Wagner, M. Drechsler, and M. Ballauff, Soft Matter 6, 1125 (2010).

[26] S. Sacanna, W. T. M. Irvine, L. Rossi, and D. J. Pine, Soft Matter 7, 1631 (2011).

[27] S. J. Gerbode, U. Agarwal, D. C. Ong, C. M. Liddell, F. Escobedo, and I. Cohen, Phys. Rev. Lett. 105, 078301 (2010).

[28] P. N. Pusey and W. van Megen, Nature (London) 320, 340 (1986).

[29] W. H. Evers, B. de Nijs, L. Filion, S. Castillo, M. Dijkstra, and D. Vanmaekelbergh, Nano Lett. 10, 4235 (2010).

[30] A. Brańka and K. W. Wojciechowski, Mol. Phys. 78, 1513 (1993).

[31] E. Widom, J. Chem. Phys. 39, 2808 (1963).

[32] D. Frenkel and B. Smit, Understanding Molecular Simulation, 2nd ed. (Academic, New York, 2001). 
[33] J. M. Polson, E. Trizac, S. Pronk, and D. Frenkel, J. Chem. Phys. 112, 5339 (2000).

[34] C. Vega and E. Noya, J. Chem. Phys. 127, 154113 (2007).

[35] J. F. Nagle, Phys. Rev. 152, 190 (1966).
[36] C. Vega and P. Monson, J. Chem. Phys. 107, 2696 (1997).

[37] M. Hoffmann, Y. Lu, M. Schrinner, M. Ballauff, and L. Harnau, J. Phys. Chem. B 112, 14843 (2008).

[38] M. Hoffmann, C. S. Wagner, L. Harnau, and A. Wittemann, ACS Nano 3, 3326 (2009). 